\title{
Vaginal Perforation, CTCAE 5.0
}

National Cancer Institute

\section{Source}

National Cancer Institute. Vaginal Perforation, CTCAE 5.0. NCI Thesaurus. Code C146697.

A disorder characterized by a rupture in the vaginal wall. 